\title{
Synthesis and evaluation of antimicrobial activity of a new series of bis(isoxazoline) derivatives
}

\author{
B. Jayashankara and K. M. Lokanatha Rai* \\ Department of studies in chemistry, University of Mysore, Manasagangothri, \\ Mysore-570006, India \\ E-mail:kmlrai@yahoo.com
}

\begin{abstract}
A series of ether-linked bis(isoxazoline) derivatives 4 were prepared by 1,3-dipolar cycloaddition reactions of nitrile oxides with allyl alcohol and allyl ethers. Products $\mathbf{4}$ were characterized by IR, NMR, and elemental analysis and were evaluated for their antimicrobial activity.
\end{abstract}

Keywords: Bis-isoxazoline, 1,3-dipolar cycloaddition, chloramine-T, antimicrobial activity

\section{Introduction}

Among five-membered heterocycles, isoxazolines represent a class of compounds of great biological importance. For instance, isoxazolines posses a broad spectrum of biological activity $^{1,2}$ (insecticidal, antibacterial, antibiotic, antitumour, antifungal, etc). Isoxazoline also serves as an important building block for the synthesis of biologically active molecules ${ }^{2}$ and serves as a prodrug for an antiarthritic agent. ${ }^{3}$ In fact, Valdecoxib is an isoxazoline derivatives, now widely used in the market as an anti-inflammatory drug. ${ }^{4}$ Literature studies reveal that bisheterocycles bearing isoxazoline ${ }^{5}$ or pyrazoline ${ }^{6}$ were synthesized via 1,3-dipolar cycloaddition of aldoxime / aldehyde hydrazone to divinyl ketone / sulfone using chloramine-T as a dehydrating agent.

1,3-Dipolar cycloaddition reactions are useful tools for the construction of biologically potent five-membered heterocycles, ${ }^{2}$ and nitrile oxides serve as excellent 1,3-dipoles. Cycloaddition of nitrile oxide to olefinic compounds are of synthetic interest, since the resulting isoxazolines are versatile intermediates for the synthesis of bifunctional compounds. ${ }^{7}$ Nitrile oxides can be generated by dehydrogenation of aryl aldoximes with mercuric acetae, ${ }^{8}$ manganese dioxide, ${ }^{9}$ tert-butyl hypochlorite, ${ }^{10}$ chloramine- ${ }^{11}$ etc. In our laboratory, ${ }^{11}$ we used chloramine-T extensively for the generation of nitrile oxides and nitrile imines from aldoximes and aldehyde hydrazones, respectively. Recently, Rai et al. used chloramine-T for the generation and cycloaddition of $\alpha$-nitrosoolefin ${ }^{12}$ and $\alpha$-azoalkenes ${ }^{13}$ from ketoximes and ketone hydrazones, 
respectively. With this background, it was considered worthwhile to prepare bis(isoxazoline) derivatives starting from simple allyl alcohols and screen them for antimicrobial activity. The present communication reports on the synthesis of ether-linked bis(isoxazoline) derivatives via 1,3-dipolar cycloaddition reactions and the evaluation of their antimicrobial activity.

\section{Results and Discussion}

\section{Synthesis}

The starting materials, aldoximes 1 were prepared from the corresponding aldehydes employing known methods. ${ }^{14}$ Oxidative dehydrogenation of aromatic aldoximes $\mathbf{1 a - d}$ by chloramine-T afforded nitrile oxides, which were intercepted in situ by allyl alcohol in refluxing ethanol. The pale yellow oils obtained were identified by NMR spectroscopy as (3-aryl-4,5-dihydroisoxazol5-yl)methanols 2a-d (Scheme 1).

Compound 2a exhibits a multiplet at $\delta 4.92-5.10$ assigned to 5-H of the isoxazoline ring. Two doublets of doublet at $\delta 3.25$ and $\delta 3.57$ correspond to $4-\mathrm{CH}_{\mathrm{A}} \mathrm{H}_{\mathrm{B}}$. A multiplet at $\delta 3.88-3.98$ and a broad signal at $\delta 3.12$ correspond to the $\mathrm{CH}_{2} \mathrm{OH}$ moiety. Thus, the formation of 2 indicates a regioselective reaction.

By stirring the hydroxylmethyl compounds 2 with excess of allyl bromide in the presence of tetrabutylammonium bromide (used as phase transfer catalyst) and potassium hydroxide, 5-allyloxymethyl-4,5-dihydroisoxazoles 3a-d were prepared as yellow oils. Compound 3a exhibits two multiplets at $\delta$ 5.26-5.37 and at $\delta$ 5.65-5.81 corresponding to the vinylic $\mathrm{CH}_{2}$ and $\mathrm{CH}$ groups, respectively. Two multiplets at $\delta 3.71-3.82$ and 4.16-4.28 correspond to the $\mathrm{CH}_{2} \mathrm{O}$ and allylic $\mathrm{CH}_{2} \mathrm{O}$ groups, respectively.

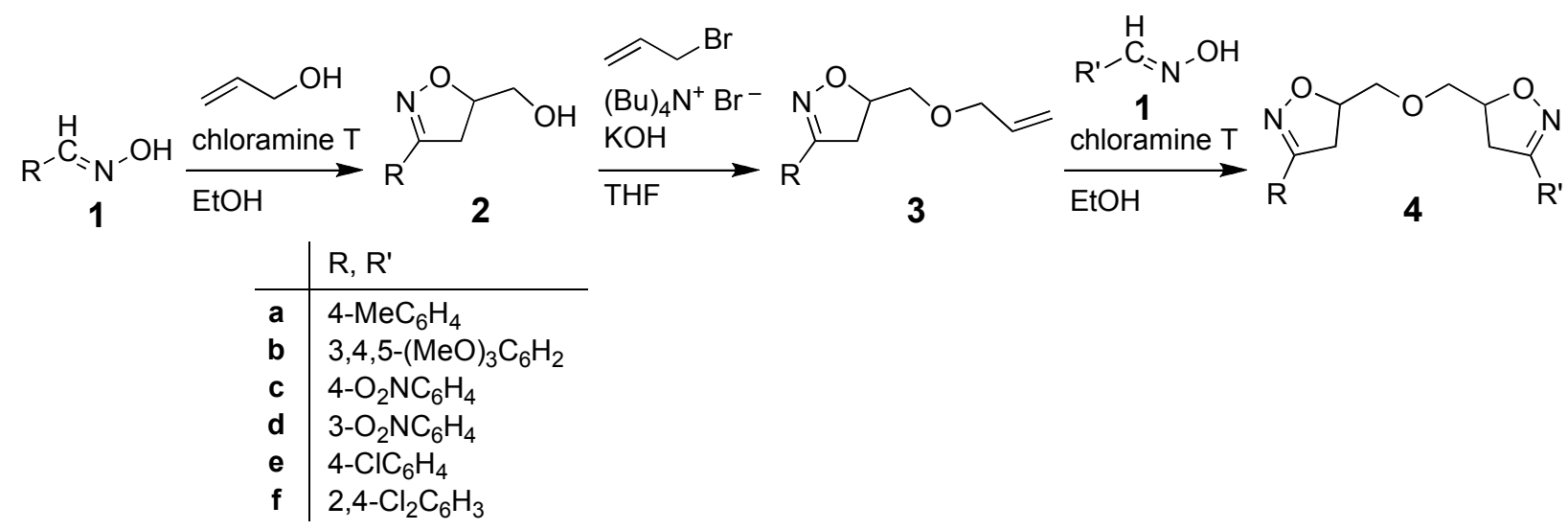

\section{Scheme 1}

The olefinic group in $\mathbf{3}$ was used to form another isoxazoline ring. Refluxing of $\mathbf{3 a}-\mathbf{d}$ with 1a-f in the presence of chloramine-T for $3 \mathrm{~h}$ gave yellow oils of 3-aryl-substituted 5-[[(4,5- 
dihydroisoxazol-5-yl)methoxy]methyl]-4,5-dihydroisoxazoles 4, characterized by IR, NMR, and elemental analyses. In addition to the aromatic and substituent signals, the ${ }^{1} \mathrm{H}$ NMR spectrum of the cycloadduct $\mathbf{4 a b}$ exhibits signals of the isoxazoline $\mathrm{CH}_{2}$ and methine groups at $\delta 3.26-3.55$ and at $\delta 4.93-5.10$, respectively; the $\mathrm{OCH}_{2}$ signals are displayed at $\delta 3.8-4.1$. The ${ }^{13} \mathrm{C} \mathrm{NMR}$ of 4ab exhibits the signals of the $\mathrm{CH}$ and $\mathrm{CH}_{2}$ groups in the isoxazoline ring at $\delta 37.7$ and at $\delta 75.5$, respectively; the $\mathrm{OCH}_{2}$ carbons resonate at 78.9. The IR confirms the absence of $\mathrm{C}=\mathrm{C}$ and $\mathrm{OH}$ groups.

\section{Antimicrobial activity}

Compounds 4 were tested for antimicrobial activity against various strains. As standards the following were used: streptomycin and tetracycline against bacteria, and Nystatin against fungi. All tests were performed in triplicate, and the average is reported. Five bacteria and five fungal species were used as the antimicrobial test strains namely: Bacillus substilis, Escherichia coli, Pseudomonas fluorescens, Xanthomonas campestris pvs, Xanthomonas oryzae, Aspergillus niger, Aspergillus flavus, Fusarium oxysporium, Trichoderma species, Fusarium and monaliforme. The bacterial strains were maintained on the LB agar medium and the filamentous fungi were maintained on potato dextrose agar (PDA) medium at $28{ }^{\circ} \mathrm{C}$. The agar disk diffusion method ${ }^{15}$ was used to test antimicrobial activity using potato dextrose agar medium.

\section{Conclusions}

Ether-linked bis(isoxazoline) derivatives 4 were synthesized and tested for antimicrobial activity by disc diffusion and micro dilution methods, showing moderate to potent inhibition.

\section{Experimental Section}

\section{Chemistry}

General Procedures. ${ }^{1} \mathrm{H}$ NMR spectra were recorded on a Bruker AM $300 \mathrm{MHz}$ spectrometer using $\mathrm{CDCl}_{3}$ as solvent and tetramethylsilane as internal standard. ${ }^{13} \mathrm{C}$ NMR spectra were measured on Jeol 400 (100 MHz) instrument. Elemental analyses were obtained on a Vaio-EL instrument. Thinlayer chromatography (TLC) was carried out with pre-coated silica gel G plates.

\section{[4,5-Dihydro-3-(p-tolyl)isoxazol-5-yl]methanol (2a). Typical procedure}

A mixture of $1 \mathrm{a}(1.0 \mathrm{~g}, 7.40 \mathrm{mmol})$ and chloramine-T trihydrate $(2.08 \mathrm{~g}, 7.41 \mathrm{mmol})$ in ethanol $(20 \mathrm{~mL})$ was stirred at room temperature for $5 \mathrm{~min}$. To this mixture, allyl alcohol $(0.43 \mathrm{~g}, 7.41$ $\mathrm{mmol})$ in ethanol $(5 \mathrm{~mL})$ was added and the reaction mixture was heated on a water bath for $3 \mathrm{~h}$. After completion of the reaction (monitored by TLC) the reaction mixture was cooled to room temperature. Sodium chloride formed was filtered off and washed with ethanol $(15 \mathrm{~mL})$. Filtrate 
and washing were combined and evaporated in vacuum. The residue was extracted with ether (25 $\mathrm{mL})$, the ether extract was washed successively with water $(2 \times 15 \mathrm{~mL}), 5 \% \mathrm{NaOH}(2 \times 15 \mathrm{~mL})$, and saturated brine solution $(10 \mathrm{~mL})$. The organic layer was dried over anhydrous sodium sulphate. After evaporation of the solvent the product was purified by the column chromatography using the chloroform/acetone (9:1) as eluent, and a yellow oil 2a was obtained (1.07 g, 76\%). ${ }^{1} \mathrm{H}$ NMR (300 MHz, $\left.\mathrm{CDCl}_{3}\right): \delta 2.34\left(\mathrm{~s}, 3 \mathrm{H}, \mathrm{CH}_{3}\right), 3.12$ (br, $\left.1 \mathrm{H}, \mathrm{OH}\right), 3.25(\mathrm{dd}, J=$ 8.0, $\left.2.0 \mathrm{~Hz}, 1 \mathrm{H}, 4-\mathrm{CH}_{\mathrm{A}}\right), 3.57\left(\mathrm{dd}, J=8.0,2.0 \mathrm{~Hz}, 1 \mathrm{H}, 4-\mathrm{CH}_{\mathrm{B}}\right), 3.88-3.98\left(\mathrm{~m}, 2 \mathrm{H}, \mathrm{OCH}_{2}\right), 4.92-$ $5.10(\mathrm{~m}, 1 \mathrm{H}, 5-\mathrm{CH}), 7.28-7.69\left(\mathrm{~m} \mathrm{4H}, \mathrm{H}_{\mathrm{Ar}}\right) .{ }^{13} \mathrm{C} \mathrm{NMR}\left(100 \mathrm{MHz}, \mathrm{CDCl}_{3}\right): \delta 24.7\left(\mathrm{CH}_{3}\right), 37.4$ $\left(\mathrm{CH}_{2}\right), 70.9\left(\mathrm{CH}_{2}\right), 77.5(\mathrm{CH}), 129.2-129.4(4 \mathrm{CH}), 131.4(\mathrm{C}), 141.3(\mathrm{C}), 156.8(\mathrm{C})$. Anal. Calcd. for $\mathrm{C}_{11} \mathrm{H}_{13} \mathrm{NO}_{2}$ : C, 69.09; H, 6.85; N, 7.32. Found: C, 69.15, H, 6.93, N, 7.30.

[4,5-Dihydro-3-(3,4,5-trimethoxyphenyl)isoxazol-5-yl]methanol (2b). From $1 \mathrm{~b}$ (1.0 g, 4.74 mmol), chloramine-T (1.33 g, $4.74 \mathrm{mmol})$ and allyl alcohol $(0.275,4.74 \mathrm{mmol})$ : Yellow oil $\mathbf{2 b}$ (0.97 g, 76\%), $R_{f} 0.32$ (chloroform/acetone, 9:1). ${ }^{1} \mathrm{H}$ NMR (300 MHz, $\left.\mathrm{CDCl}_{3}\right): \delta 3.14(\mathrm{br}, 1 \mathrm{H}$, $\mathrm{OH}), 3.23\left(\mathrm{dd}, J=8.0,4.0 \mathrm{~Hz}, 1 \mathrm{H}, 4-\mathrm{CH}_{\mathrm{A}}\right), 3.56\left(\mathrm{dd}, J=8.0,4.0 \mathrm{~Hz}, 1 \mathrm{H}, 4-\mathrm{CH}_{\mathrm{B}}\right), 3.85-3.96(\mathrm{~m}$, $\left.2 \mathrm{H}, \mathrm{OCH}_{2}\right), 3.90\left(\mathrm{~s}, 6 \mathrm{H}, \mathrm{OCH}_{3}\right), 3.93\left(\mathrm{~s}, 3 \mathrm{H}, \mathrm{OCH}_{3}\right), 4.98-5.10(\mathrm{~m}, 1 \mathrm{H}, 5-\mathrm{CH}), 6.83(\mathrm{~s}, 2 \mathrm{H}$, $\left.\mathrm{H}_{\mathrm{Ar}}\right) .{ }^{13} \mathrm{C} \mathrm{NMR}\left(100 \mathrm{MHz}, \mathrm{CDCl}_{3}\right): \delta 36.7\left(\mathrm{CH}_{2}\right), 56.4\left(2 \mathrm{OCH}_{3}\right), 56.6\left(\mathrm{OCH}_{3}\right), 70.6\left(\mathrm{CH}_{2}\right), 77.2$ $(\mathrm{CH}), 106.7$ (2 CH), 128.2 (C), $141.3(\mathrm{C}), 150.8$ (2C) $156.1(\mathrm{C})$. Anal. Calcd. for $\mathrm{C}_{13} \mathrm{H}_{17} \mathrm{NO}_{5}$ : C, 58.42; H, 6.41; N, 5.24. Found: C, 58.44; H, 6.45, N, 5.22.

[4,5-Dihydro-3-(4-nitrophenyl)isoxazol-5-yl]methanol (2c). From 1c (1.0 g, $6.02 \mathrm{mmol})$, chloramine-T (1.70 g, $6.04 \mathrm{mmol})$ and allyl alcohol $(0.35 \mathrm{~g}, 6.03 \mathrm{mmol})$ : Yellow oil $2 \mathrm{c}(0.95 \mathrm{~g}$, $71 \%) R_{f} 0.32$ (chloroform/acetone, 9:1). ${ }^{1} \mathrm{H}$ NMR (300 MHz, $\mathrm{CDCl}_{3}$ ): $\delta 3.19$ (br, $\left.1 \mathrm{H}, \mathrm{OH}\right), 3.29$ $\left(\mathrm{dd}, J=8.0,3.0 \mathrm{~Hz}, 1 \mathrm{H}, 4-\mathrm{CH}_{\mathrm{A}}\right), 3.60\left(\mathrm{dd}, J=8.0,3.0 \mathrm{~Hz}, 1 \mathrm{H}, 4-\mathrm{CH}_{\mathrm{B}}\right), 3.87-3.95(\mathrm{~m}, 2 \mathrm{H}$, $\left.\mathrm{OCH}_{2}\right), 5.09-5.21(\mathrm{~m}, 1 \mathrm{H}, 5-\mathrm{CH}), 7.80-8.19\left(4 \mathrm{H}, \mathrm{H}_{\mathrm{Ar}}\right) .{ }^{13} \mathrm{C} \mathrm{NMR}\left(100 \mathrm{MHz}, \mathrm{CDCl}_{3}\right): \delta 37.2$ $\left(\mathrm{CH}_{2}\right), 70.6\left(\mathrm{CH}_{2}\right), 77.4(\mathrm{CH}), 121.3(2 \mathrm{CH}), 130.2(2 \mathrm{CH}), 140.3(\mathrm{C}), 150.9(\mathrm{C}), 156.4(\mathrm{C})$. Anal. Calcd. for $\mathrm{C}_{10} \mathrm{H}_{10} \mathrm{~N}_{2} \mathrm{O}_{4}$ : C, 54.05; H, 4.54; N, 12.61. Found: C, 54.02; H, 4.55, N, 12.62.

[4,5-Dihydro-3-(3-nitrophenyl)isoxazol-5-yl]methanol (2d). From 1d (1.0 g, $6.02 \mathrm{mmol})$, chloramine-T $(1.70 \mathrm{~g}, 6.04 \mathrm{mmol})$ and allyl alcohol $(0.35 \mathrm{~g}, 6.03 \mathrm{mmol})$ : yellow oil $2 \mathrm{~d}(0.9 \mathrm{~g}$, 67\%), $R_{f} 0.36$ (chloroform/acetone, 9:1). ${ }^{1} \mathrm{H}$ NMR (300 MHz, $\mathrm{CDCl}_{3}$ ): $\delta 3.18$ (br, $\left.1 \mathrm{H}, \mathrm{OH}\right), 3.28$ $\left(\mathrm{dd}, J=8.0,4.0 \mathrm{~Hz}, 1 \mathrm{H}, 4-\mathrm{CH}_{\mathrm{A}}\right), 3.61\left(\mathrm{dd}, J=8.0,3.0 \mathrm{~Hz}, 1 \mathrm{H}, 4-\mathrm{CH}_{\mathrm{B}}\right), 3.89-3.99(\mathrm{~m}, 2 \mathrm{H}$, $\left.\mathrm{OCH}_{2}\right), 5.11-5.20(\mathrm{~m}, 1 \mathrm{H}, 5-\mathrm{CH}), 7.67-8.60\left(\mathrm{~m}, 4 \mathrm{H}, \mathrm{H}_{\mathrm{Ar}}\right) .{ }^{13} \mathrm{C} \mathrm{NMR}\left(100 \mathrm{MHz}, \mathrm{CDCl}_{3}\right): \delta 37.1$ $\left(\mathrm{CH}_{2}\right), 70.4\left(\mathrm{CH}_{2}\right), 77.2(\mathrm{CH}), 123.3(\mathrm{CH}), 124.1(\mathrm{CH}), 129.9(\mathrm{CH}), 134.7(\mathrm{C}), 135.3(\mathrm{CH})$, 148.6 (C), 156.5 (C). Anal. Calcd. for $\mathrm{C}_{10} \mathrm{H}_{10} \mathrm{~N}_{2} \mathrm{O}_{4}$ : C, 54.05; H, 4.54; N, 12.61. Found: C, 54.02; $\mathrm{H}, 4.55, \mathrm{~N}, 12.62$.

\section{5-[(Allyloxy)methyl]-4,5-dihydro-3-(p-tolyl)isoxazole (3a). Typical procedure}

A mixture of $2 \mathrm{a}(1.0 \mathrm{~g}, 5.23 \mathrm{mmol})$, allyl bromide $(0.63 \mathrm{~g}, 5.25 \mathrm{mmol})$, tetrabutylammonium bromide $(0.17 \mathrm{~g}, 0.53 \mathrm{mmol})$ and potassium hydroxide $(0.29 \mathrm{~g}, 5.18)$ was stirred overnight in THF (15 mL). After completion of the reaction (monitered by TLC), the solvent was evaporated under vacuum, the residue was extracted with ethyl acetate $(2 \times 15 \mathrm{~mL})$, washed with water $(2 \mathrm{x}$ $10 \mathrm{~mL})$ and dried $\left(\mathrm{Na}_{2} \mathrm{SO}_{4}\right)$. The solvent was evaporated, and the residue was subjected to 
column chromatography (chloroform/benzene, 8:2) to give a yellow oil 3a $(0.98 \mathrm{~g}, 81 \%) .{ }^{1} \mathrm{H}$ NMR (300 MHz, $\left.\mathrm{CDCl}_{3}\right): \delta 2.38\left(\mathrm{~s}, 3 \mathrm{H}, \mathrm{CH}_{3}\right), 3.23\left(\mathrm{dd}, J=8.0,4.0 \mathrm{~Hz}, 1 \mathrm{H}, 4-\mathrm{CH}_{\mathrm{A}}\right), 3.55(\mathrm{dd}, J$ $\left.=8.0,3.0 \mathrm{~Hz}, 1 \mathrm{H}, 4-\mathrm{CH}_{\mathrm{B}}\right), 3.71-3.82\left(\mathrm{~m}, 2 \mathrm{H}, \mathrm{OCH}_{2}\right), 4.16-4.28\left(\mathrm{~m}, 2 \mathrm{H}, \mathrm{OCH}_{2}\right), 4.98-5.10(\mathrm{~m}$, $1 \mathrm{H}, 5-\mathrm{CH}), 5.26-5.37\left(\mathrm{~m}, 2 \mathrm{H},=\mathrm{CH}_{2}\right), 5.65-5.81(\mathrm{~m}, 1 \mathrm{H},=\mathrm{CH}), 7.12-7.45\left(\mathrm{~m}, 4 \mathrm{H}, \mathrm{H}_{\mathrm{Ar}}\right) .{ }^{13} \mathrm{C}$ NMR (100 MHz, $\left.\mathrm{CDCl}_{3}\right): \delta 24.7\left(\mathrm{CH}_{3}\right), 37.7\left(\mathrm{CH}_{2}\right), 74.9\left(\mathrm{CH}_{2}\right), 75.5(\mathrm{CH}), 78.9\left(\mathrm{CH}_{2}\right), 115.8$ $\left(\mathrm{CH}_{2}\right), 129.1-129.3$ (4 CH), $131.2(\mathrm{C}), 141.2(\mathrm{C}), 151.7(\mathrm{CH}), 156.6(\mathrm{C})$. Anal. Calcd. for $\mathrm{C}_{14} \mathrm{H}_{17} \mathrm{NO}_{2}$ : C, 72.70; H, 7.41; N, 6.06. Found: C, 72.72, H, 7.40, N, 6.07.

5-[(Allyloxy)methyl]-4,5-dihydro-3-(3,4,5-trimethoxyphenyl)isoxazole (3b). From $2 \mathrm{~b}$ (1.0 g, $3.74 \mathrm{mmol})$, allyl bromide $(0.45 \mathrm{~g}, 3.75 \mathrm{mmol})$ and tetrabutylammonium bromide $(0.12 \mathrm{~g}, 0.37$ mmol) and potassium hydroxide $(0.21 \mathrm{~g}, 3.75 \mathrm{mmol})$ : Pale yellow oil $(0.84 \mathrm{~g}, 73 \%) R_{f} 0.47$ (chloroform/benzene, 8:2). ${ }^{1} \mathrm{H}$ NMR $\left(300 \mathrm{MHz} \mathrm{CDCl}_{3}\right): \delta 3.22\left(\mathrm{dd}, J=8.0,3.0 \mathrm{~Hz}, 1 \mathrm{H}, 4-\mathrm{CH}_{\mathrm{A}}\right.$ ), $3.54\left(\mathrm{dd}, J=9.0,3.0 \mathrm{~Hz}, 1 \mathrm{H}, 4-\mathrm{CH}_{\mathrm{B}}\right), 3.68-3.81\left(\mathrm{~m}, 2 \mathrm{H}, \mathrm{OCH}_{2}\right), 3.88\left(\mathrm{~s}, 6 \mathrm{H}, \mathrm{OCH}_{3}\right), 3.93(\mathrm{~s}$, $\left.3 \mathrm{H}, \mathrm{OCH}_{3}\right), 4.10-4.26\left(\mathrm{~m}, 2 \mathrm{H}, \mathrm{OCH}_{2}\right), 4.97-5.08(\mathrm{~m}, 1 \mathrm{H}, 5-\mathrm{CH}), 5.21-5.34\left(\mathrm{~m}, 2 \mathrm{H},=\mathrm{CH}_{2}\right)$, 5.61-5.77 (m, 1H, =CH), $6.84\left(\mathrm{~s}, 2 \mathrm{H}, \mathrm{H}_{\mathrm{Ar}}\right) .{ }^{13} \mathrm{C} \mathrm{NMR}(100 \mathrm{MHz}, \mathrm{CDCl} 3): \delta 37.5\left(\mathrm{CH}_{2}\right), 56.3(2$ $\left.\mathrm{OCH}_{3}\right), 56.6\left(\mathrm{OCH}_{3}\right), 74.8\left(\mathrm{CH}_{2}\right), 75.5(\mathrm{CH}), 78.7\left(\mathrm{CH}_{2}\right), 115.7\left(\mathrm{CH}_{2}\right), 106.6(2 \mathrm{CH}), 128.2(\mathrm{C})$, 131.4 (C), 141.4 (C), 151.3 (2C), 156.4 (C). Anal. Calcd. for $\mathrm{C}_{16} \mathrm{H}_{21} \mathrm{NO}_{5}$ : C, 62.53; H, 6.89; N, 4.56. Found: C, 62.52, H, 6.84, N, 4.57 .

5-[(Allyloxy)methyl]-4,5-dihydro-3-(4-nitrophenyl)isoxazole (3c). From $2 \mathrm{c}$ (1.0 g, $4.5 \mathrm{mmol})$, allyl bromide $(0.54 \mathrm{~g}, 4.5 \mathrm{mmol})$ and tetrabutylammonium bromide $(0.15 \mathrm{~g}, 0.45 \mathrm{mmol})$ and potassium hydroxide $(0.25 \mathrm{~g}, 4.55 \mathrm{mmol})$ : Pale yellow oil 3c $(0.82 \mathrm{~g}, 70 \%) R_{f} 0.46$ (chloroformbenzene, 8:2). ${ }^{1} \mathrm{H}$ NMR (300 MHz, $\left.\mathrm{CDCl}_{3}\right): \delta 3.27\left(\mathrm{dd}, J=8.0-2.0 \mathrm{~Hz}, 1 \mathrm{H}, 4-\mathrm{CH}_{\mathrm{A}}\right), 3.56(\mathrm{dd}, J$ $\left.=8.0,2.0 \mathrm{~Hz}, 1 \mathrm{H}, 4-\mathrm{CH}_{\mathrm{B}}\right), 3.70-3.83\left(\mathrm{~m}, 2 \mathrm{H}, \mathrm{OCH}_{2}\right), 4.13-4.28\left(\mathrm{~m}, 2 \mathrm{H}, \mathrm{OCH}_{2}\right), 5.08-5.18(\mathrm{~m}$, $1 \mathrm{H}, 5-\mathrm{CH}), 5.25-5.35\left(\mathrm{~m}, 2 \mathrm{H},=\mathrm{CH}_{2}\right), 5.64-5.79(\mathrm{~m}, 1 \mathrm{H},=\mathrm{CH}), 7.78-8.21\left(\mathrm{~m}, 4 \mathrm{H}, \mathrm{H}_{\mathrm{Ar}}\right) .{ }^{13} \mathrm{C}$ NMR (100 MHz, $\left.\mathrm{CDCl}_{3}\right): \delta 37.7\left(\mathrm{CH}_{2}\right), 74.9\left(\mathrm{CH}_{2}\right), 75.6(\mathrm{CH}), 78.8\left(\mathrm{CH}_{2}\right), 115.9\left(\mathrm{CH}_{2}\right), 121.3$ $(2 \mathrm{CH}), 130.2(2 \mathrm{C}), 131.8(\mathrm{C}), 140.4(\mathrm{C}), 151.5(\mathrm{C}), 156.5(\mathrm{C})$. Anal. Calcd. for $\mathrm{C}_{13} \mathrm{H}_{14} \mathrm{~N}_{2} \mathrm{O}_{4}$ : C, 59.54; H, 5.38; N, 10.68. Found;: C, 59.56, H, 5.36 N, 10.69.

5-[(Allyloxy)methyl]-4,5-dihydro-3-(3-nitrophenyl)isoxazole (3d). From 2d (1.0 g, $4.5 \mathrm{mmol})$, allyl bromide $(0.54 \mathrm{~g}, 4.5 \mathrm{mmol})$ and tetrabutylammonium bromide $(0.15 \mathrm{~g}, 0.45 \mathrm{mmol})$ and potassium hydroxide $(0.25 \mathrm{~g}, 4.55 \mathrm{mmol})$ as pale yellow oil in $(0.8 \mathrm{~g}, 68 \%$ yield $) R_{f} 0.50$ (chloroform-benzene, 8:2). ${ }^{1} \mathrm{H}$ NMR $\left(300 \mathrm{MHz}, \mathrm{CDCl}_{3}\right.$ ): $\delta 3.27$ (dd, $J=7.0,2.0 \mathrm{~Hz}, 1 \mathrm{H}, 4-$ $\left.\mathrm{CH}_{\mathrm{A}}\right), 3.56\left(\mathrm{dd}, J=8.0,2.0,1 \mathrm{H}, 4-\mathrm{CH}_{\mathrm{B}}\right), 3.70-3.83\left(\mathrm{~m}, 2 \mathrm{H}, \mathrm{OCH}_{2}\right), 4.13-4.28\left(\mathrm{~m}, 2 \mathrm{H}, \mathrm{OCH}_{2}\right)$, 5.07-5.18 (m, 1H, CH), 5.25-5.35 (m, 2H, $\left.=\mathrm{CH}_{2}\right), 5.64-5.79(\mathrm{~m}, 1 \mathrm{H},=\mathrm{CH}), 7.68-8.31(\mathrm{~m}, 4 \mathrm{H}$, $\left.\mathrm{H}_{\mathrm{Ar}}\right) \cdot{ }^{13} \mathrm{C}$ NMR $\left(100 \mathrm{MHz}, \mathrm{CDCl}_{3}\right): \delta 37.7\left(\mathrm{CH}_{2}\right), 74.9\left(\mathrm{CH}_{2}\right), 75.6(\mathrm{CH}), 78.8\left(\mathrm{CH}_{2}\right), 115.9$ $\left(\mathrm{CH}_{2}\right), 121.3(2 \mathrm{CH}), 130.2(2 \mathrm{C}), 131.8(\mathrm{C}), 140.4(\mathrm{C}), 151.5(\mathrm{C}), 156.5(\mathrm{C})$; Anal. Calcd. for $\mathrm{C}_{13} \mathrm{H}_{14} \mathrm{~N}_{2} \mathrm{O}_{4}$ : C, 59.54; H, 5.38; N, 10.68. Found: C, 59.56, H, 5.36 N, 10.69.

\section{5-[[(4,5-Dihydro-3-(3,4,5-trimethoxyphenyl)isoxazol-5-yl)methoxy]methyl]-4,5-dihydro-3-} (p-tolyl)isoxazole (4ab). Typical procedure

A mixture of $\mathbf{3 a}(1.0 \mathrm{~g}, 4.33 \mathrm{mmol}), \mathbf{1 b}(0.91 \mathrm{~g}, 4.31 \mathrm{mmol})$ and chloramine-T trihydrate $(1.22 \mathrm{~g}$, $4.34 \mathrm{mmol})$ in ethanol $(20 \mathrm{~mL})$ was heated on a water bath. After $3 \mathrm{~h}$ the reaction was 
completed, and the mixture was cooled to room temperature. Sodium chloride formed was filtered off and washed with ethanol $(15 \mathrm{~mL})$. Filtrate and washing were combined and the solvent was evaporated in vacuum. The residue was extracted with ether $(25 \mathrm{~mL})$, the extract was washed successively with water $(2 \times 15 \mathrm{~mL}), 10 \% \mathrm{NaOH}(2 \times 15 \mathrm{~mL})$, and saturated brine solution $(10 \mathrm{~mL})$. The organic layer was dried over anhydrous sodium sulphate. Evaporation of the solvent yielded the product, which was subjected to column chromatography using chloroform as eluent to give a yellow oil $4 \mathbf{4 a b}(1.34 \mathrm{~g}, 68 \%), R_{f} 0.65$ (chloroform). ${ }^{1} \mathrm{H}$ NMR (300 $\left.\mathrm{MHz}, \mathrm{CDCl}_{3}\right): \delta 2.37\left(\mathrm{~s}, 3 \mathrm{H}, \mathrm{CH}_{3}\right), 3.26-3.55\left(\mathrm{~m}, 4 \mathrm{H}, 4-, 4^{\prime}-\mathrm{CH}_{2}\right), 3.8-4.1\left(\mathrm{~m}, 4 \mathrm{H}, \mathrm{OCH}_{2}\right), 3.90$ $\left(\mathrm{s}, 6 \mathrm{H}, \mathrm{OCH}_{3}\right), 3.95\left(\mathrm{~s}, 3 \mathrm{H}, \mathrm{OCH}_{3}\right), 4.93-5.10(\mathrm{~m}, 2 \mathrm{H}, 5-, 5$ ' $-\mathrm{CH}), 6.90\left(\mathrm{~s}, 2 \mathrm{H}, \mathrm{H}_{\mathrm{Ar}}\right), 7.26-7.67$ $\left(\mathrm{m}, 4 \mathrm{H}, \mathrm{H}_{\mathrm{Ar}}\right) \cdot{ }^{13} \mathrm{C}$ NMR $\left(100 \mathrm{MHz}, \mathrm{CDCl}_{3}\right): \delta 24.7\left(\mathrm{CH}_{3}\right), 37.7\left(2 \mathrm{CH}_{2}\right), 56.2\left(2 \mathrm{OCH}_{3}\right), 56.5$ $\left(\mathrm{OCH}_{3}\right), 75.5(2 \mathrm{CH}), 78.9\left(2 \mathrm{CH}_{2}\right), 106.9(2 \mathrm{CH}), 128.6(\mathrm{C}), 129.2-129.4(4 \mathrm{CH}), 131.2(\mathrm{C})$, 141.1 (C), 141.7 (C), 151.3 (2 C), 156.9 (2 C). Anal. Calcd. for $\mathrm{C}_{24} \mathrm{H}_{28} \mathrm{~N}_{2} \mathrm{O}_{6}$ : C, 65.44; $\mathrm{H}, 6.41$; N, 6.36. Found: C, 65.45, H, 6.43, N, 6.33.

\section{5-[[[4,5-Dihydro-3-(4-nitrophenyl)isoxazol-5-yl]methoxy]methyl]-4,5-dihydro-3-(p-tolyl)-} isoxazole (4ac). From 3a $(0.5 \mathrm{~g}, 2.16 \mathrm{mmol}), 1 \mathrm{c}(0.36 \mathrm{~g}, 2.16 \mathrm{mmol})$ and chloramine- $\mathrm{T}$ trihydrate $(0.62 \mathrm{~g}, 2.20 \mathrm{mmol})$ : Yellow oil $4 \mathrm{ac}(0.61 \mathrm{~g}, 69 \%), R_{f} 0.60$ (chloroform). ${ }^{1} \mathrm{H}$ NMR (300 MHz, $\left.\mathrm{CDCl}_{3}\right): \delta 2.37$ (s, 3H, $\left.\mathrm{CH}_{3}\right), 3.20-3.55$ (m, 4H, 4-, 4'- $\mathrm{CH}_{2}$ ), 3.8-4.1 (m, 4H, $\mathrm{OCH}_{2}$ ), $5.0\left(\mathrm{~m}, 2 \mathrm{H}, 5-, 5{ }^{\prime}-\mathrm{CH}\right), 7.24-8.11\left(\mathrm{~m}, 8 \mathrm{H}, \mathrm{H}_{\mathrm{Ar}}\right),{ }^{13} \mathrm{C} \mathrm{NMR}\left(100 \mathrm{MHz}, \mathrm{CDCl}_{3}\right): \delta 24.5\left(\mathrm{CH}_{3}\right)$, $37.5\left(2 \mathrm{CH}_{2}\right), 75.3(2 \mathrm{CH}), 78.7\left(2 \mathrm{CH}_{2}\right), 121.7(2 \mathrm{CH}), 129.2-129.3(4 \mathrm{CH}), 129.6(2 \mathrm{CH}), 131.1$ (C), 140.3 (C), 140.9 (C), 151.1 (C), 156.8 (2C). Anal. Calcd. for $\mathrm{C}_{21} \mathrm{H}_{21} \mathrm{~N}_{3} \mathrm{O}_{5}$ : C, 63.79; $\mathrm{H}$, $5.35 ; \mathrm{N}, 10.63$. Found: C, 63.73, H, 5.39, N, 10.53 .

\section{5-[[[4,5-dihydro-3-(p-tolyl)isoxazol-5-yl]methoxy]methyl]-3-(4-chlorophenyl)-4,5-dihydro} isoxazole (4ae). From 3a $(0.5 \mathrm{~g}, 2.16 \mathrm{mmol})$, $1 \mathrm{e}(0.34 \mathrm{~g}, 2.19 \mathrm{mmol})$ and chloramine-T trihydrate $(0.62 \mathrm{~g}, 2.20 \mathrm{mmol})$ : Yellow oil 4ae $(0.66 \mathrm{~g}, 76 \%), R_{f} 0.67$ (chloroform). ${ }^{1} \mathrm{H}$ NMR $\left(300 \mathrm{MHz}, \mathrm{CDCl}_{3}\right): \delta 2.36\left(\mathrm{~s}, 3 \mathrm{H}, \mathrm{CH}_{3}\right), 3.3-3.65\left(\mathrm{~m}, 4 \mathrm{H}, 4-, 4^{\prime}-\mathrm{CH}_{2}\right), 3.75-4.05(\mathrm{~m}, 4 \mathrm{H}$, $\left.\mathrm{OCH}_{2}\right), 5.0\left(\mathrm{~m}, 2 \mathrm{H}, 5-, 5\right.$ '-CH), 7.22-7.97 (m, 8H, $\left.\mathrm{H}_{\mathrm{Ar}}\right) .{ }^{13} \mathrm{C} \mathrm{NMR}\left(100 \mathrm{MHz}, \mathrm{CDCl}_{3}\right): \delta 24.4$ $\left(\mathrm{CH}_{3}\right), 37.4\left(2 \mathrm{CH}_{2}\right), 75.2(2 \mathrm{CH}), 78.7\left(2 \mathrm{CH}_{2}\right), 129.0-129.2(4 \mathrm{CH}), 129.3-130.7(4 \mathrm{CH}), 131.2$ (C), 132.3 (C), 136. 8 (C), 140.8 (C), 156.6 (2C). Anal. Calcd. for $\mathrm{C}_{21} \mathrm{H}_{21} \mathrm{ClN}_{2} \mathrm{O}_{3}$ : C, 65.54; $\mathrm{H}$, 5.50 ; N, 7.28. Found: C, 65.53, H, 5.49, N, 7.34.

5-[[[4,5-Dihydro-3-(p-tolyl)isoxazol-5-yl]methoxy]methyl]-3-(2,4-dichlorophenyl)-4,5-dihydroisoxazole (4af). From 3a $(0.5 \mathrm{~g}, 2.16 \mathrm{mmol})$, 1f $(0.41 \mathrm{~g}, 2.16 \mathrm{mmol})$ and chloramine- $\mathrm{T}$ trihydrate $(0.62 \mathrm{~g}, 2.20 \mathrm{mmol})$ : Yellow oil 4 af $(0.76 \mathrm{~g}, 72 \%), R_{f} 0.64$ (chloroform). ${ }^{1} \mathrm{H}$ NMR $\left(300 \mathrm{MHz}, \mathrm{CDCl}_{3}\right): \delta 2.34\left(\mathrm{~s}, 3 \mathrm{H}, \mathrm{CH}_{3}\right), 3.35-3.65\left(\mathrm{~m}, 4 \mathrm{H}, 4-, 4^{\prime}-\mathrm{CH}_{2}\right), 3.72-3.97(\mathrm{~m}, 4 \mathrm{H}$, $\left.\mathrm{OCH}_{2}\right), 5.1\left(\mathrm{~m}, 2 \mathrm{H}, 5-, 5^{\prime}-\mathrm{CH}\right), 7.20-8.13\left(\mathrm{~m}, 7 \mathrm{H}, \mathrm{H}_{\mathrm{Ar}}\right) .{ }^{13} \mathrm{C} \mathrm{NMR}\left(100 \mathrm{MHz}, \mathrm{CDCl}_{3}\right): \delta 24.4$ $\left(\mathrm{CH}_{3}\right), 37.7\left(2 \mathrm{CH}_{2}\right), 75.5(2 \mathrm{CH}), 78.9\left(2 \mathrm{CH}_{2}\right), 127.1(\mathrm{CH}), 129.1-129.3(4 \mathrm{CH}), 130.7(\mathrm{CH})$, 131.1 (C), $132.3(\mathrm{CH}), 135.5$ (C), 135.7 (C), 137.8 (C), 140.7 (C), 156.5 (2C). Anal. Calcd. for $\mathrm{C}_{21} \mathrm{H}_{20} \mathrm{Cl}_{2} \mathrm{~N}_{2} \mathrm{O}_{3}$ : C, 60.15; H, 4.81; N, 6.68. Found: C, 60.16, H, 4.79, N, 6.71.

5-[[[4,5-Dihydro-3-(3,4,5-trimethoxyphenyl)isoxazol-5-yl]methoxy]methyl]-4,5-dihydro-3(4-nitrophenyl)isoxazole (4bc): From 3b $(0.5 \mathrm{~g}, 1.62 \mathrm{mmol}), 1 \mathrm{c}(0.27 \mathrm{~g}, 1.62 \mathrm{mmol})$ and chloramine-T trihydrate $(0.46 \mathrm{~g}, 1.63 \mathrm{mmol})$ : Yellow oil $4 \mathrm{bc}(0.56 \mathrm{~g}, 73 \%), R_{f} 0.53$ (chloroform). ${ }^{1} \mathrm{H}$ NMR (300 MHz, $\mathrm{CDCl}_{3}$ ): $\delta 3.15-3.51\left(\mathrm{~m}, 4 \mathrm{H}, 4-,{ }^{\prime}-\mathrm{CH}_{2}\right), 3.63-3.74\left(\mathrm{~m}, 4 \mathrm{H}, \mathrm{OCH}_{2}\right.$ ), 
$3.88\left(\mathrm{~s}, 6 \mathrm{H}, \mathrm{OCH}_{3}\right), 3.92\left(\mathrm{~s}, 3 \mathrm{H}, \mathrm{OCH}_{3}\right), 5.10(\mathrm{~m}, 2 \mathrm{H}, 5-, 5$ '- $\mathrm{CH}), 6.8\left(\mathrm{~s}, 2 \mathrm{H}, \mathrm{H}_{\mathrm{Ar}}\right), 7.70-8.3(\mathrm{~m}$, $\left.4 \mathrm{H}, \mathrm{H}_{\mathrm{Ar}}\right) .{ }^{13} \mathrm{C} \mathrm{NMR}\left(100 \mathrm{MHz}, \mathrm{CDCl}_{3}\right): \delta 37.3\left(2 \mathrm{CH}_{2}\right), 56.3\left(2 \mathrm{OCH}_{3}\right), 56.6\left(\mathrm{OCH}_{3}\right), 75.2(2$ $\mathrm{CH}), 78.5\left(2 \mathrm{CH}_{2}\right), 106.8(2 \mathrm{CH}), 121.3(2 \mathrm{CH}), 128.4(\mathrm{C}), 130.2(2 \mathrm{CH}), 140.2(\mathrm{C}), 141.6(\mathrm{C})$, 150.7 (C), 150.9 (2C), 156.9 (2C). Anal. Calcd. for $\mathrm{C}_{23} \mathrm{H}_{25} \mathrm{~N}_{3} \mathrm{O}_{8}$ : C, 58.59; H, 5.34; N, 8.91. Found: C, 58.62, H, 5.35, N, 8.98.

5-[[[3-(4-Chlorophenyl)-4,5-dihydroisoxazol-5-yl]methoxy]methyl]-4,5-dihydro-3-(3,4,5-trimethoxyphenyl)isoxazole (4be): Obtained from $3 \mathbf{b}(0.5 \mathrm{~g}, 1.62 \mathrm{mmol})$, 1 e $(0.25 \mathrm{~g}, 1.61 \mathrm{mmol})$ and chloramine-T trihydrate $(0.46 \mathrm{~g}, 1.63 \mathrm{mmol})$ : Yellow oil 4be $(0.5 \mathrm{~g}, 67 \%), R_{f} 0.60$ (chloroform). ${ }^{1} \mathrm{H}$ NMR (300 MHz, $\mathrm{CDCl}_{3}$ ): $\delta 3.24-3.57\left(\mathrm{~m}, 4 \mathrm{H}, 4-, 4{ }^{\prime}-\mathrm{CH}_{2}\right), 3.68-3.78(\mathrm{~m}, 4 \mathrm{H}$, $\left.\mathrm{OCH}_{2}\right), 3.89\left(\mathrm{~s}, 6 \mathrm{H}, \mathrm{OCH}_{3}\right), 3.93\left(\mathrm{~s}, 3 \mathrm{H}, \mathrm{OCH}_{3}\right), 5.20(\mathrm{~m}, 2 \mathrm{H}, 5-, 5$ ' $-\mathrm{CH}), 6.70\left(\mathrm{~s}, 2 \mathrm{H}, \mathrm{H}_{\mathrm{Ar}}\right)$, 7.27-7.60 (m, 4H, $\left.\mathrm{H}_{\mathrm{Ar}}\right) .{ }^{13} \mathrm{C} \mathrm{NMR}\left(100 \mathrm{MHz}, \mathrm{CDCl}_{3}\right): \delta 37.5\left(2 \mathrm{CH}_{2}\right), 56.3\left(2 \mathrm{OCH}_{3}\right), 56.6$ $\left(\mathrm{OCH}_{3}\right), 75.3(2 \mathrm{CH}), 78.8\left(2 \mathrm{CH}_{2}\right), 106.8(2 \mathrm{CH}), 128.4(\mathrm{C}), 129.1(2 \mathrm{CH}), 130.4(2 \mathrm{CH}), 132.1$ (C), 136.7 (C), 141.3 (C), 150.8 (2 C), 156.84 (2 C). Anal. Calcd. for $\mathrm{C}_{23} \mathrm{H}_{25} \mathrm{ClN}_{2} \mathrm{O}_{2}$ : C, 59.94; $\mathrm{H}$, 5.47; N, 6.08. Found: C, 59.95, H, 5.49, N, 6.03.

5-[I[3-(2,4-Dichlorophenyl)-4,5-dihydroisoxazol-5-yl]methoxy]methyl]-4,5-dihydro-3-(3,4,5trimethoxyphenyl)isoxazole (4bf). From $3 \mathrm{~b}(0.5 \mathrm{~g}, 1.62 \mathrm{mmol}), \mathbf{1 f}(0.31 \mathrm{~g}, 1.63 \mathrm{mmol})$, and chloramine-T trihydrate $(0.46 \mathrm{~g}, 1.63 \mathrm{mmol})$ : Yellow oil $4 \mathbf{b f}(0.59 \mathrm{~g}, 73 \%), R_{f} 0.58$ (chloroform). ${ }^{1} \mathrm{H}$ NMR $\left(300 \mathrm{MHz}, \mathrm{CDCl}_{3}\right): \delta 3.25-3.60\left(\mathrm{~m}, 4 \mathrm{H}, 4-, 4{ }^{\prime}-\mathrm{CH}_{2}\right), 3.63-3.75\left(\mathrm{~m}, 4 \mathrm{H}, \mathrm{OCH}_{2}\right), 3.86$ $\left(\mathrm{s}, 6 \mathrm{H}, \mathrm{OCH}_{3}\right), 3.90\left(\mathrm{~s}, 3 \mathrm{H}, \mathrm{OCH}_{3}\right), 5.0-5.3\left(\mathrm{~m}, 2 \mathrm{H}, 5-, 5\right.$ '-CH), $6.70\left(\mathrm{~s}, 2 \mathrm{H}, \mathrm{H}_{\mathrm{Ar}}\right), 7.2-7.5(\mathrm{~m}$, $3 \mathrm{H}, \mathrm{H}) .{ }^{13} \mathrm{C} \mathrm{NMR}\left(100 \mathrm{MHz}, \mathrm{CDCl}_{3}\right): \delta 37.6\left(2 \mathrm{CH}_{2}\right), 56.3\left(2 \mathrm{OCH}_{3}\right), 56.6\left(\mathrm{OCH}_{3}\right), 75.2(2 \mathrm{CH})$, $78.7\left(2 \mathrm{CH}_{2}\right), 106.7(2 \mathrm{CH}), 127.1(\mathrm{CH}), 128.3(\mathrm{C}), 130.5(\mathrm{CH}), 132.2(\mathrm{CH}), 135.2(\mathrm{C}), 135.4$ (C), 138.7 (C), 141.4 (C), 150.8 (2 C), 156.4 (2 C). Anal. Calcd. for $\mathrm{C}_{23} \mathrm{H}_{24} \mathrm{Cl}_{2} \mathrm{~N}_{2} \mathrm{O}_{6}$ : C, 55.77; $\mathrm{H}$, 4.88; N, 5.66. Found: C, 55.75, H, 4.89, N, 5.63.

5-[[[4,5-Dihydro-3-(4-nitrophenyl)isoxazol-5-yl]methoxy]methyl]-3-(4-chlorophenyl)-4,5-dihydroisoxazole (4ce). From 3c $(0.5 \mathrm{~g}, 1.90 \mathrm{mmol})$, $1 \mathrm{e}(0.3 \mathrm{~g}, 1.93 \mathrm{mmol})$, and chloramine-T trihydrate (0.55 g, $1.96 \mathrm{mmol})$ : Yellow oil 4ce $(0.5 \mathrm{~g}, 64 \%), R_{f} 0.62$ (chloroform). ${ }^{1} \mathrm{H}$ NMR (300 $\mathrm{MHz}_{\mathrm{CDCl}}$ ): $\delta 3.38-3.58\left(\mathrm{~m}, 4 \mathrm{H}, 4-, 4^{\prime}-\mathrm{CH}_{2}\right), 3.78-3.98\left(\mathrm{~m}, 4 \mathrm{H}, \mathrm{OCH}_{2}\right), 5.13-5.20(\mathrm{~m}, 2 \mathrm{H}, 5-$, 5'-CH), 7.34-8.16 (m, 8H, H $\left.{ }_{\mathrm{Ar}}\right) .{ }^{13} \mathrm{C} \mathrm{NMR}\left(100 \mathrm{MHz}, \mathrm{CDCl}_{3}\right): \delta 37.4\left(2 \mathrm{CH}_{2}\right), 75.2(2 \mathrm{CH}), 78.7$ $\left(2 \mathrm{CH}_{2}\right), 121.3(2 \mathrm{CH}), 129.2(2 \mathrm{CH}), 130.2(2 \mathrm{CH}), 130.7(2 \mathrm{CH}), 132.1(\mathrm{C}), 136.6(\mathrm{C}), 140.2$ (C), 150.8 (C), 156.6 (2 C). Anal. Calcd. for $\mathrm{C}_{20} \mathrm{H}_{18} \mathrm{ClN}_{3} \mathrm{O}_{5}$ : C, 57.77; H, 4.36; N, 10.11. Found: C, 57.79, H, 4.38, N, 10.13.

5-[I[4,5-Dihydro-3-(4-nitrophenyl)isoxazol-5-yl]methoxy]methyl]-3-(2,4-dichlorophenyl)4,5-dihydroisoxazole (4cf). From 3c (0.5 g, $1.90 \mathrm{mmol})$, 1f (0.36 g, $1.89 \mathrm{mmol})$, and chloramine-T trihydrate $(0.55 \mathrm{~g}, 1.96 \mathrm{mmol})$ : Yellow oil $4 \mathbf{c f}(0.56 \mathrm{~g}, 65 \%), R_{f} 0.67$ (chloroform). ${ }^{1} \mathrm{H}$ NMR (300 MHz, $\left.\mathrm{CDCl}_{3}\right): \delta 3.41-3.59\left(\mathrm{~m}, 4 \mathrm{H}, 4-, 4{ }^{\prime}-\mathrm{CH}_{2}\right), 3.82-3.98\left(\mathrm{~m}, 4 \mathrm{H}, \mathrm{OCH}_{2}\right), 5.15-$ $5.21\left(\mathrm{~m}, 2 \mathrm{H}, 5-, 5^{\prime}-\mathrm{CH}\right), 7.21-7.73\left(\mathrm{~m}, 4 \mathrm{H}, \mathrm{H}_{\mathrm{Ar}}\right), 7.92-8.16\left(\mathrm{~m}, 3 \mathrm{H}, \mathrm{H}_{\mathrm{Ar}}\right) .{ }^{13} \mathrm{C} \mathrm{NMR}(100 \mathrm{MHz}$, $\left.\mathrm{CDCl}_{3}\right): \delta 36.4\left(\mathrm{CH}_{2}\right), 37.4(\mathrm{CH}), 75.2(2 \mathrm{CH}), 78.6\left(2 \mathrm{CH}_{2}\right), 121.2(2 \mathrm{CH}), 127.1(\mathrm{CH}), 130.2$ $(2 \mathrm{CH}), 130.5(\mathrm{CH}), 132.1(\mathrm{CH}), 135.2(\mathrm{C}), 135.4(\mathrm{C}), 138.2(\mathrm{C}), 140.2(\mathrm{C}), 150.7(\mathrm{C}), 156.3$ (C). 156.7 (C). Anal. Calcd. for $\mathrm{C}_{20} \mathrm{H}_{17} \mathrm{C}_{12} \mathrm{~N}_{3} \mathrm{O}_{5}$ : C, 53.35; H, 3.81; N, 9.33. Found: C, 53.39, H, $3.80, \mathrm{~N}, 9.35$.

5-[[[4,5-Dihydro-3-(p-tolyl)isoxazol-5-yl]methoxy]methyl]-3-(3-nitrophenyl)-4,5-dihydrois- 
oxazole (4da). From 3d $(0.5 \mathrm{~g}, 1.90 \mathrm{mmol})$, $1 \mathrm{a}(0.26 \mathrm{~g}, 1.92 \mathrm{mmol})$, and chloramine-T trihydrate (0.55 g, $1.96 \mathrm{mmol})$ : Yellow oil 4da $(0.59 \mathrm{~g}, 78 \%), R_{f} 0.68$ (chloroform). ${ }^{1} \mathrm{H}$ NMR (300 MHz, $\left.\mathrm{CDCl}_{3}\right): \delta 2.37\left(\mathrm{~s}, 3 \mathrm{H}, \mathrm{CH}_{3}\right), 3.23-3.49\left(\mathrm{~m}, 4 \mathrm{H}, 4-, 4{ }^{\prime}-\mathrm{CH}_{2}\right), 3.76-3.89\left(\mathrm{~m}, 4 \mathrm{H}, \mathrm{OCH}_{2}\right), 4.98-$ $5.12\left(\mathrm{~m}, 2 \mathrm{H}, 5-, 5^{\prime}-\mathrm{CH}\right), 7.12-7.56\left(\mathrm{~m}, 4 \mathrm{H}, \mathrm{H}_{\mathrm{Ar}}\right), 7.62-8.33\left(\mathrm{~m}, 4 \mathrm{H}, \mathrm{H}_{\mathrm{Ar}}\right) .{ }^{13} \mathrm{C} \mathrm{NMR}(100 \mathrm{MHz}$, $\left.\mathrm{CDCl}_{3}\right): \delta 24.4\left(\mathrm{CH}_{3}\right), 37.4\left(2 \mathrm{CH}_{2}\right), 75.2(2 \mathrm{CH}), 78.8\left(2 \mathrm{CH}_{2}\right), 123.5(\mathrm{CH}), 124.2(\mathrm{CH}), 129.1-$ $129.3(4 \mathrm{CH}), 129.8(\mathrm{CH}), 131.1(\mathrm{C}), 134.8(\mathrm{C}), 135.3(\mathrm{CH}), 140.5(\mathrm{C}), 148.7(\mathrm{C}), 156.9(2 \mathrm{C}$ Anal. Calcd. for $\mathrm{C}_{21} \mathrm{H}_{21} \mathrm{~N}_{3} \mathrm{O}_{5}$ : C, 63.79; H, 5.35; N, 10.63. Found; C, 63.75, H, 5.37, N, 10.66.

5-[[[4,5-Dihydro-3-(3,4,5-trimethoxyphenyl)-5-yl]methoxy]methyl]-3-(3-nitrophenyl)-4,5-

dihydroisoxazole (4db). From 3d $(0.5 \mathrm{~g}, 1.90 \mathrm{mmol}), \mathbf{1 b}(0.4 \mathrm{~g}, 1.90 \mathrm{mmol})$, and chloramine-T trihydrate (0.54 g, $1.92 \mathrm{mmol})$ : Yellow oil $4 \mathrm{db}(0.63 \mathrm{~g}, 70 \%), R_{f} 0.65$ (chloroform). ${ }^{1} \mathrm{H}$ NMR (300 MHz, $\left.\mathrm{CDCl}_{3}\right): \delta 3.23-3.55\left(\mathrm{~m}, 4 \mathrm{H}, 4-, 4{ }^{\prime}-\mathrm{CH}_{2}\right), 3.62-3.81\left(\mathrm{~m}, 4 \mathrm{H}, \mathrm{OCH}_{2}\right), 3.90(\mathrm{~s}, 6 \mathrm{H}$, $\left.\mathrm{OCH}_{3}\right), 3.94\left(\mathrm{~s}, 3 \mathrm{H}, \mathrm{OCH}_{3}\right), 5.02-5.32\left(\mathrm{~m}, 2 \mathrm{H}, 5-, 5\right.$ '-CH), $6.80\left(\mathrm{~s}, 2 \mathrm{H}, \mathrm{H}_{\mathrm{Ar}}\right), 7.63-8.46(\mathrm{~m}, 4 \mathrm{H}$, $\left.\mathrm{H}_{\mathrm{Ar}}\right) .{ }^{13} \mathrm{C} \mathrm{NMR}\left(100 \mathrm{MHz}, \mathrm{CDCl}_{3}\right): \delta 37.5\left(2 \mathrm{CH}_{2}\right), 56.2\left(2 \mathrm{OCH}_{3}\right), 56.5\left(\mathrm{OCH}_{3}\right), 75.4(2 \mathrm{CH})$, $78.7\left(2 \mathrm{CH}_{2}\right), 106.9(2 \mathrm{CH}), 123.6(\mathrm{CH}), 124.3(\mathrm{CH}), 128.5(\mathrm{C}), 129.8(\mathrm{CH}), 134.9(\mathrm{C}), 135.3$ $(\mathrm{CH}), 141.6(\mathrm{C}), 148.7$ (C), 151.3 (2 C), 156.9 (2 C). Anal. Calcd. for $\mathrm{C}_{23} \mathrm{H}_{25} \mathrm{~N}_{3} \mathrm{O}_{8}$ : C, 58.59; $\mathrm{H}$, 5.34; N, 8.91. Found; C, 58.45, H, 5.33, N, 8.93.

\section{Microbiology}

Table 1. Minimal inhibitory concentration $\mathrm{X}\left[\mu \mathrm{g} \mathrm{mL}^{-1}\right]$ and inhibitory zone diameter $\mathrm{Y}[\mathrm{mm}]$ of compounds 4 against tested bacterial strains

\begin{tabular}{|c|c|c|c|c|c|c|c|c|c|c|}
\hline \multirow[t]{2}{*}{ Compound } & \multicolumn{2}{|c|}{$\begin{array}{l}\text { Bacillus } \\
\text { substilis }\end{array}$} & \multicolumn{2}{|c|}{ Escherichia coli } & \multicolumn{2}{|c|}{$\begin{array}{c}\text { Pseudomonas } \\
\text { fluorescens }\end{array}$} & \multicolumn{2}{|c|}{$\begin{array}{l}\text { Xanthomonas } \\
\text { campestris pvs }\end{array}$} & \multicolumn{2}{|c|}{$\begin{array}{c}\text { Xanthomonas } \\
\text { oryzae }\end{array}$} \\
\hline & $\mathrm{X}$ & $\mathrm{Y}$ & $\mathrm{X}$ & $\mathrm{Y}$ & $X$ & $\mathrm{Y}$ & $X$ & $\mathrm{Y}$ & $\mathrm{X}$ & $\mathrm{Y}$ \\
\hline $4 a b$ & 32 & 7 & 35 & 7 & 31 & 9 & 34 & 6 & 28 & 7 \\
\hline $4 a c$ & 28 & 6 & 32 & 6 & 28 & 6 & 31 & 6 & 27 & 6 \\
\hline $4 a e$ & 27 & 9 & 31 & 6 & 28 & 4 & 29 & 5 & 26 & 9 \\
\hline 4af & 22 & 11 & 26 & 8 & 23 & 10 & 25 & 7 & 21 & 10 \\
\hline $4 \mathrm{bc}$ & 22 & 10 & 22 & 10 & 26 & 11 & 23 & 11 & 24 & 12 \\
\hline 4be & 25 & 7 & 22 & 9 & 28 & 13 & 27 & 10 & 23 & 11 \\
\hline $4 \mathrm{bf}$ & 21 & 7 & 26 & 13 & 23 & 16 & 25 & 12 & 23 & 12 \\
\hline $4 c e$ & 22 & 10 & 21 & 11 & 25 & 14 & 22 & 11 & 24 & 11 \\
\hline $4 \mathrm{cf}$ & 19 & 13 & 17 & 14 & 20 & 17 & 19 & 14 & 23 & 12 \\
\hline $4 d a$ & 29 & 4 & 27 & 10 & 32 & 10 & 24 & 10 & 26 & 9 \\
\hline $4 d b$ & 24 & 9 & 22 & 9 & 29 & 15 & 25 & 12 & 23 & 10 \\
\hline Streptomycin & 19 & 13 & 13 & 14 & 12 & 17 & - & - & - & - \\
\hline Tetracycline & - & - & - & - & - & - & 9 & 12 & 13 & 12 \\
\hline
\end{tabular}

Streptomycin sulfate (25 $\mu \mathrm{g}$ per disc) and Tetracycline $(25 \mu \mathrm{g}$ per disc) were used as positive reference standard antibacterial discs; compounds 4 (25 $\mu \mathrm{g}$ per disc). 
The bacteria inoculum was prepared by suspending in $9 \mathrm{~mL}$ of sterile water for colonies from a $24 \mathrm{~h}$ culture on LB agar medium. For the filamentous fungi, the inoculum was prepared with the spores derived from 5-15 days cultures on PDA medium. The mycelia were covered with 10 $\mathrm{mL}$ of distilled water and the conidia were scraped using sterile pipette. The spores were recovered after filtration on sterile absorbent cotton and were resuspended in sterile distilled water. The cell density of each inoculum was adjusted with hemocytometer in order to obtain a final concentration of approximately $10^{4}$ to $10^{6} \mathrm{CFU} \mathrm{mL}{ }^{-1}$ for the bacteria and filomentous fungi, respectively.

Table 2. Minimal inhibitory concentration $X\left[\mu \mathrm{g} \mathrm{mL}^{-1}\right]$ and inhibitory zone diameter $\mathrm{Y}[\mathrm{mm}]$ of compounds 4 against tested fungal strains

\begin{tabular}{|c|c|c|c|c|c|c|c|c|c|c|}
\hline \multirow[t]{2}{*}{ Compound } & \multicolumn{2}{|c|}{$\begin{array}{c}\text { Aspergillus } \\
\text { niger }\end{array}$} & \multicolumn{2}{|c|}{$\begin{array}{c}\text { Aspergillus } \\
\text { flavus }\end{array}$} & \multicolumn{2}{|c|}{$\begin{array}{c}\text { Fusarium } \\
\text { oxysporium }\end{array}$} & \multicolumn{2}{|c|}{$\begin{array}{c}\text { Fusarium } \\
\text { monalifome }\end{array}$} & \multicolumn{2}{|c|}{$\begin{array}{c}\text { Trichoderma } \\
\text { species }\end{array}$} \\
\hline & $X$ & $\mathrm{Y}$ & $\mathrm{X}$ & $\mathrm{Y}$ & $X$ & $\mathrm{Y}$ & $\mathrm{X}$ & $\mathrm{Y}$ & $X$ & $\mathrm{Y}$ \\
\hline $4 a b$ & 22 & 4 & 26 & 5 & 23 & 8 & 17 & 7 & 23 & 8 \\
\hline $4 a c$ & 24 & 4 & 24 & 8 & 21 & 6 & 19 & 6 & 26 & 7 \\
\hline $4 a e$ & 27 & 5 & 27 & 6 & 20 & 6 & 16 & 6 & 25 & 9 \\
\hline 4af & 19 & 6 & 21 & 8 & 18 & 9 & 14 & 9 & 19 & 11 \\
\hline $4 \mathrm{bcc}$ & 18 & 8 & 18 & 9 & 14 & 12 & 14 & 11 & 15 & 14 \\
\hline $4 \mathrm{be}$ & 18 & 7 & 18 & 7 & 18 & 11 & 15 & 9 & 17 & 12 \\
\hline $4 \mathrm{bf}$ & 16 & 8 & 16 & 10 & 13 & 13 & 11 & 11 & 14 & 15 \\
\hline 4ce & 17 & 7 & 16 & 8 & 15 & 11 & 13 & 10 & 16 & 13 \\
\hline $4 \mathrm{cf}$ & 15 & 9 & 15 & 11 & 11 & 14 & 10 & 12 & 13 & 16 \\
\hline $4 \mathrm{da}$ & 18 & 6 & 19 & 6 & 18 & 7 & 15 & 7 & 19 & 10 \\
\hline $4 d b$ & 16 & 7 & 18 & 9 & 13 & 12 & 12 & 10 & 14 & 13 \\
\hline Nystatin & 15 & 8 & 15 & 10 & 11 & 14 & 9 & 12 & 12 & 16 \\
\hline
\end{tabular}

Nystatin ( $25 \mu \mathrm{g}$ per disc) was used as positive reference standard antifungal discs; compounds 4 (25 $\mu \mathrm{g}$ per disc).

Nystatin (Himedia) was used as a positive control for fungi, and streptomycin and tetracycline for bacteria. Each disk contained $25 \mu \mathrm{g}$ of standard drugs and $25 \mu \mathrm{g}$ of compound 4. Plates were first kept at $4{ }^{\circ} \mathrm{C}$ for at least $2 \mathrm{~h}$ to allow the diffusion of chemicals and then incubated at $28{ }^{\circ} \mathrm{C}$. Inhibition zone were measured after $24 \mathrm{~h}$ of incubation of bacteria and after $48 \mathrm{~h}$ of incubation for fungi.

The micro dilution method ${ }^{16}$ was used to evaluate the minimum inhibitory concentration (MIC) of compounds 4. The nutrient liquid medium and potato dextrose liquid medium were used as test media. Tests were performed in 96-well round bottom sterile culture plates. The suspensions of yeast and filamentous fungi were adjusted in sterile water to match the density of a 0.5 McFarland standard. The wells of a micro dilution plates were inoculated with $180 \mathrm{~mL}$ of 
the culture medium containing a final inoculum of $0.5-2.5 \times 10^{3} \mathrm{CFU} \mathrm{mL}^{-1}$. All compounds were dissolved in DMSO and were diluted twofold in the liquid medium to give a range of concentration from 640 to $0.1 \mu \mathrm{g} \mathrm{mL}^{-1} .20 \mu \mathrm{L}$ of each concentration were added to wells containing culture suspension except the growth control well. The final concentration ranged from 64 to $0.01 \mu \mathrm{g} \mathrm{mL}{ }^{-1}$. Plates were incubated at $35{ }^{\circ} \mathrm{C}$ for $48 \mathrm{~h}$. Fungi growth was assessed at $494 \mathrm{~nm}$ by measuring the optical density in each well using an enzyme immunoassay multiwell reader (Sigma Diagnostic).

\section{Acknowledgements}

We wish to acknowledge financial support from the University of Mysore, and M. Suresh Babu for providing the spectral data.

\section{References}

1. Huisgen, R. Angew. Chem. Int. Ed.1963, 2, 565.

2. Caramella, P.; Gruenanger, P. In 1,3-Dipolar Cycloaddition Chemistry, Vol. 1, Padwa, A., Ed.; Wiley Interscience: New York, 1984; p337.

3. Patterson, J. W.; Cheung, P. S.; Ernest, M. J. J. Med. Chem. 1992, 35, 507.

4. Dannhardt, G.; Kiefer, W.; Kramer, G.; Maehrlein, S.; Nowe, U.; Fiebich, B. Eur. J. Med. Chem. 2000, 35, 499.

5. Padmavathi, V.; Reddy, B. Jagan Mohan; Reddy, B. Chandra Obula; Padmaja, A. Tetrahedron 2005, 61, 2407.

6. Padmavathi, V.; Reddy, K. Venugopal; Padmaja, A.; Bhaskar, D. Reddy Phosphorus, Sulfur, Silicon 2003, 178, 171.

7. Umesha, K. B.; Kumar, K. Ajay; Rai, K. M. Lokanatha Synth. Commun. 2002, 32, 1841.

8. Rai, K. M. Lokanatha; Linganna, N.; Hassner, A.; Anjanamurthy, C. Org. Prep. Proc. Int. 1992, 24, 91.

9. Keigiel, J.; Poplawaska, M.; Jozwik, J.; Kosior, M.; Jurzak, J. Tetrahedron 1999, 40, 5605.

10. Moriya, O.; Takenaka, H.; Iyoda, M.; Urata, Y.; Endo, T. J. Chem. Soc., Perkin Trans I. 1994, 413.

11. (a) Rai, K. M. Lokanatha; Hassner, A. Indian J. Chem. 1997, 36B, 242. (b) Rai, K. M. Lokanatha; Hassner, A. Synth. Commun. 1997, 27, 467. (c) Hassner, A.; Rai, K. M. Lokanatha Synthesis 1989, 57. (d) Rai, K. M. Lokanatha; Hassner, A. Synth. Commun. 1989, 19, 2799.

12. Gaonkar, S. L.; Rai, K. M. Lokanatha J. Heterocycl. Chem. 2005, 42, 877.

13. Gaonkar, S. L.; Rai, K. M. Lokanatha Tetrahedron Lett. 2005, 46, 5969. 
14. Vogel, A. I. A Textbook of Practical Organic Chemistry, 5th Edn.; Longman's Green Co. Ltd: London, 1989; pp 1258.

15. Lemriss, S.; Marquet, B.; Ginestet, H.; Lefeuvre, L.; Fassouane, A.; Boiron, P. J. Mycol. Med. 2003, 13, 189.

16. Zgoda, J. R.; Porter, J. R. Pharm. Biol. 2001, 39, 221. 